\title{
Producción científica en diabetes en Perú: Un estudio bibliométrico
}

\author{
Scientific production in diabetes in Peru: A bibliometric study
}

\begin{abstract}
Objective: to describe the scientific production related to diabetes in Peru between 1996 and 2015. Methods: Bibliometric study. Scientific papers on diabetes published between 1996 and 2015 were identified by authors with Peruvian affiliation in the Web of Science Core Collection (WoS) and Scopus databases. The variables of interest were extracted manually. We present descriptive results and collaborative networks. Results: We found 81 publications, $75.3 \%$ were original articles, of which none evaluated public health interventions and $60.7 \%$ received foreign funding; $55.6 \%$ had an author affiliated with the Universidad Peruana Cayetano Heredia (UPCH), and $65.4 \%$ had a foreign author. Five clusters of international collaboration networks could be distinguished, all with the participation of the UPCH. Conclusion: Peruvian scientific production related to diabetes is low, with an absence of studies evaluating public health interventions, and with collaboration networks focused on UPCH.
\end{abstract}

Keywords: Diabetes; bibliometry; collaboration networks; international collaboration; research funding.

\section{INTRODUCCIÓN}

La diabetes mellitus es un problema de salud pública creciente, de manera que el número estimado de adultos viviendo con diabetes alrededor del mundo ha aumentado de 108 millones en 1980 a 422 millones en el 2014, representando un incremento del $4,7 \%$ al $8,5 \%$ de prevalencia de diabetes en la población adulta. Este aumento es mayor en países de ingresos bajos y medios (PIBM) ${ }^{1}$, en los cuales además existen sendas dificultades para el diagnóstico y manejo de esta condición, resultando en mayor discapacidad y muerte ${ }^{2,3}$.

Por ello, los PIBM requieren con urgencia generar información local que permita implementar intervenciones orientadas a disminuir el impacto de la diabetes en términos de morbilidad y mortalidad. Sin embargo, es sabido que la producción científica biomédica en los PIBM es baja, sus instituciones investigadores son pocas, su presupuesto para investigación es escaso, y la investigación realizada en
Alvaro Taype-Rondan', Oscar Huapaya-Huertas², Guido Bendezu-Quispe², Josmel Pacheco-Mendoza3, Mayte Bryce-Alberti'

Este trabajo fue recibido el 28 de noviembre de 2016. Aceptado con modificaciones el 24 de marzo de 2017 y aceptado para su publicación el 5 de mayo de 2017.

muchas ocasiones no obedece a las necesidades locales ${ }^{4}$.

En este contexto, resulta importante caracterizar la producción científica local sobre diabetes e identificar su problemática, lo cual permitirá a las entidades gubernamentales, académicas y científicas orientar sus políticas de investigación. De esta manera, estudios bibliométricos anteriores han evaluado la producción científica en diabetes en India ${ }^{5}$, Nigeria ${ }^{6}$, Irán ${ }^{7}$, Tailandia, Argentina $^{8}$, Brasil ${ }^{9}$, y en países del Medio Oriente ${ }^{10,11}$.

En Perú, para el 2015, se estimó que la prevalencia de diabetes entre adultos de 25 años o más fue del $7,0 \%$; y que de estas personas el $40 \%$ no había sido diagnosticado, lo cual refleja un importante problema de sub-diagnóstico ${ }^{12}$. Además, un estudio realizado en hospitales del sistema de vigilancia de diabetes del Perú encontró que, entre los pacientes que tuvieron alguna consulta de control, el 73,4\% tuvo un valor de hemoglobina glicosilada mayor o igual que 
$7,0 \%{ }^{13}$, indicativo de un control inadecuado de la diabetes, posiblemente relacionado con problemas en el acceso y uso adecuado de los servicios de salud ${ }^{14}$.

Esta compleja problemática de la diabetes en Perú requiere políticas que favorezcan la investigación en diabetes. Sin embargo, a la fecha no se han publicado estudios bibliométricos en este tema, por lo cual el presente estudio tiene como objetivo describir la producción científica en diabetes en Perú entre 1996 y 2015.

\section{MATERIALES Y MÉTODOS \\ DISEÑOY BASES DE DATOS USADAS}

Estudio observacional bibliométrico, realizado en los artículos científicos publicados en las bases de datos electrónicas Web of Science Core Collection (WoS) y Scopus, entre 1996 y 2015.

Scopus incluye más de 21.500 revistas de ciencia, tecnología, ciencias sociales, artes, humanidades y medicina ${ }^{15}$. En tanto que WoS incluye 12.700 revistas científicas de diversos temas. Los estudios bibliométricos suelen usar la principal colección de revistas (core collection) ${ }^{16,17}$. Se decidió emplear estas dos bases de datos debido a la gran cantidad de revistas biomédicas que incluyen, $y$ a su riguroso proceso de selección de revistas, lo cual permite colectar los estudios más relevantes sobre el tema.

Estrategia de búsqueda

Durante julio del 2016 se realizaron búsquedas de manera sistemática en las bases de datos mencionadas. El término clave usado para las búsquedas fue "diabetes", con el cual se conoce a esta enfermedad en español, inglés y otros idiomas. Además, se buscaron solamente artículos que tengan algún autor con filiación peruana. De esta manera, la estrategia de búsqueda para Scopus fue: [AFFILCOUNTRY (peru) AND TITLE-ABS-KEY (diabetes)] y para WoS: [CU=PERU AND TS=DIABETES].

Se obtuvieron 198 resultados en Scopus y 134 resultados en WoS. Posteriormente, tres autores seleccionaron independientemente los artículos científicos que contaran por lo menos con un autor con filiación peruana y que tuvieran entre sus temas principales la diabetes (ya sea como población de estudio, como variable, o como población beneficiada). Las selecciones fueron comparadas por un cuarto autor. En caso de observarse diferencias en la selección de estudios, estas se resolvieron por consenso entre los autores. Finalmente, se obtuvieron 81 artículos que cumplieron con dichos criterios.

\section{Extracción de variables}

Tres autores leyeron independientemente los títulos, los resúmenes, y de ser necesario los manuscritos in-extenso de los artículos incluidos, y recolectaron independientemente las variables de interés en una ficha de recolección de datos, usando el software Microsoft Excel 2013. Las bases de datos fueron comparadas por un cuarto autor, y las diferencias fueron resueltas en consenso.
Se recolectaron las siguientes variables para cada artículo científico: base de datos en el que está indizada la revista (Scopus, WoS, o ambos), nombre de la revista, si la revista se encontró en el primer cuartil según su impacto en SClmago (sí o no), año de publicación, Idioma del in-extenso, colaboración extranjera (tener algún autor con filiación extranjera), filiación de los autores, filiación del autor corresponsal, número de instituciones peruanas participantes según la filiación de los autores, ubicación de las instituciones peruanas participantes (todas son de Lima, todas son de fuera de Lima, o tanto de Lima como de fuera de Lima), y tipo de artículo (original, de revisión, u otros).

Para los artículos originales (definido por tener la estructura: introducción, métodos, resultados y discusión; o una estructura similar) se determinó el diseño del estudio: transversal, longitudinal, revisión sistemática, preclínicos, (con animales o en laboratorio), o de intervención. Entre los estudios de intervención, se diferenció aquellos de intervenciones en salud pública. Además, en este grupo se evaluó el financiamiento del estudio, consignando cinco categorías: no declarar tener o no tener financiamiento, declarar haber sido autofinanciados por los autores, declarar haber sido financiados solo por fondos extranjeros, declarar haber sido financiados solo por fondos peruanos, y declarar haber sido financiados tanto por fondos peruanos como por fondos extranjeros.

Análisis estadístico

Para el análisis de colaboración, se creó una base de datos con todos los registros en el paquete de gestión de referencias Endnote v7.7, se exportó el conteo de artículos a Microsoft Excel, a partir del cual se creó una matriz de coocurrencia con la macro Toolinf, que se importó a UCINET v6.624 y se guardó como un archivo ".net", para usarlo en el graficador de redes Netdraw en el cual se realizaron los análisis de grado de centralidad y grado de intermediación para una mejor distribución de los nodos ${ }^{18}$. Los resultados fueron presentados descriptivamente mediante frecuencias absolutas y relativas, usando el programa estadístico STATA v13.0.

El resultado fue exportado al software de visualización VosViewer v1.6.5 ${ }^{19}$, en el cual se obtuvo en el gráfico con los conglomerados de colaboración, donde el tamaño de los círculos representa la cantidad de artículos y el grosor de las líneas indica el número de artículos en colaboración.

\section{RESULTADOS}

Se encontraron 81 publicaciones en revistas científicas indizadas a WoS o Scopus entre 1996 y 2015 que tuvieron entre sus temas principales a la diabetes. De estos, 76 $(93,8 \%)$ artículos estuvieron indizados a Scopus, 24 (29,6\%) artículos estuvieron indizados a WoS, y 19 (23,5\%) artículos estuvieron indizados en ambas bases de datos. De ellos, 61 trabajos $(75,3 \%)$ fueron originales, de los cuales 33 fueron transversales, 12 fueron longitudinales, 7 fueron revisiones sistemáticas, 7 fueron preclínicos y 2 fueron estudios de 
intervención, es decir, ensayos clínicos realizados con fármacos hipoglicemiantes.

Entre los 61 trabajos originales, $12(19,7 \%)$ no declaran tener o no financiamiento, $5(8,2 \%)$ declaran haber sido autofinanciados por los autores, 30 (49,2\%) declaran haber sido financiados solo por fondos extranjeros, 7 (11,5\%) declaran haber sido financiados solo por fondos peruanos y $7(11,5 \%)$ declaran haber sido financiados tanto poro fondos peruanos como por fondos extranjeros.

Se observa un incremento irregular en el número de publicaciones sobre diabetes, de manera que el 2015 se llegaron a publicar 18 artículos (Figura 1). De los 81 artículos evaluados, $39(48,1 \%)$ fueron publicados en una revista del primer cuartil (Q1) según SCImago, proporción que no pareció variar en los últimos años (Figura 2). Los artículos se publicaron en 57 revistas diferentes. La revista con más artículos publicados fue la "Revista Peruana de Medicina Experimental y Salud Pública" con quince artículos (18,5\%), seguida de "Plos One" y "Diabetes Research and Clinical Practice" con cuatro artículos $(4,9 \%)$ cada una.

Se contabilizaron 42 instituciones peruanas: 23 públicas y 19 privadas. Las instituciones que participaron con mayor número de artículos fueron la UPCH (45 artículos; 55,6\%), el Hospital Nacional Cayetano Heredia (12 artículos; 14,8\%), el Ministerio de Salud (7 artículos; 8,6\%), el Instituto Nacional de Salud ( 6 artículos; 7,4\%), y la Universidad Nacional Mayor de San Marcos (5 artículos; 6,2\%). Del total de trabajos, 44 $(54,3 \%)$ fueron realizados por una sola institución peruana
Con respecto a los autores, 15 (18,5\%) artículos tuvieron algún autor peruano con filiación fuera de la ciudad de Lima, 41 (50,6\%) tuvieron como autor corresponsal a un investigador filiación peruana, siendo la Universidad Peruana Cayetano Heredia (UPCH) la institución más común en autores corresponsales (Tabla 1). Existió participación de por lo menos un autor con filiación extranjera en 53 (65,4\%) artículos, siendo los países con más artículos en colaboración: Estados Unidos (35 artículos, 43,2\%), Brasil, Chile, Colombia, Argentina, México (12 artículos cada uno, 14,8\%), Reino Unido (11 artículos, 13,6\%) y España (10 artículos, 12,3\%).

En cuanto a las redes de colaboración, las colaboraciones con mayor número de artículos entre instituciones peruanas se dan entre la UPCH y el Hospital Nacional Cayetano Heredia, y entre la UPCH y la organización no gubernamental de desarrollo Prisma. Otras redes de colaboración nacionales tienen como centros al Instituto Nacional de Salud y al Ministerio de Salud (Figura 3a).

Al analizar todas las colaboraciones, se pueden definir cinco conglomerados: 1) UPCH, Prisma, Johns Hopkins University, y University of London; 2) UPCH con hospitales de España; 3) UPCH con universidades y hospitales europeos; 4) Ministerio de Salud del Perú y otras entidades Latinoamericanas; 5) Clínica el Golf, Universidad de Sao Paulo y otras instituciones latinoamericanas y europeas (Figura 3b).

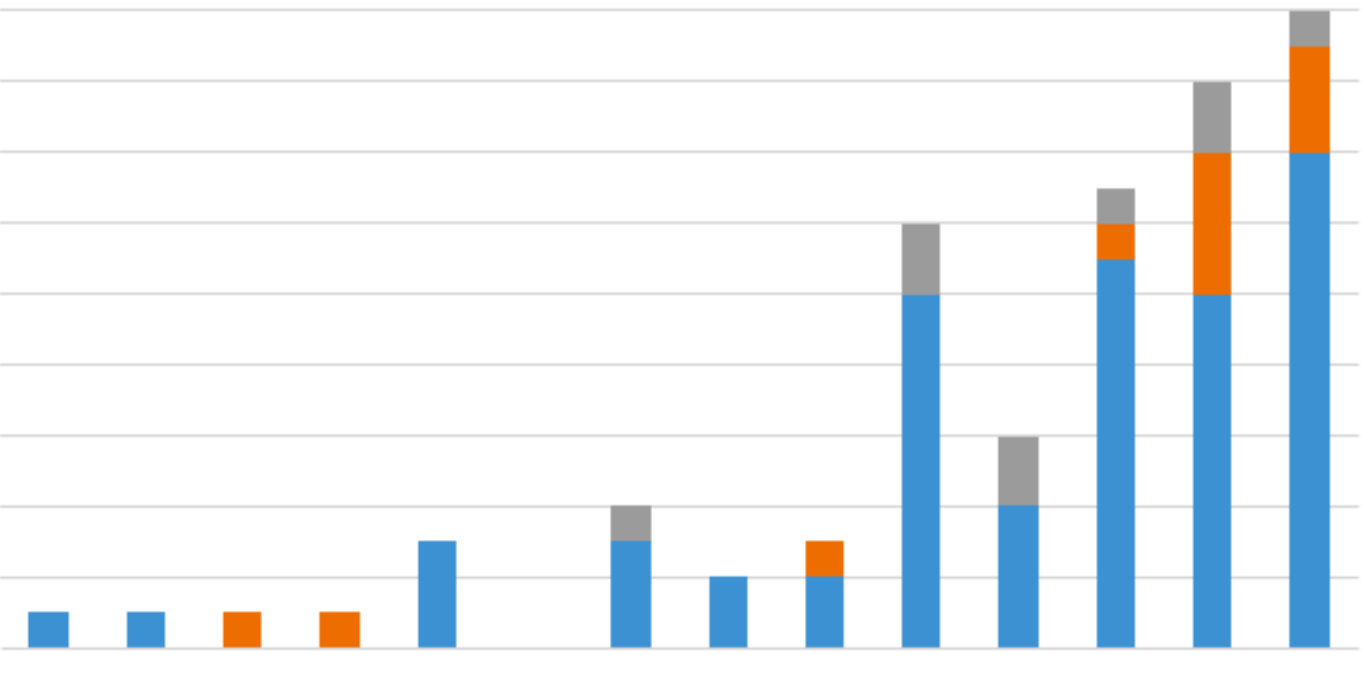

19962003200420052006200720082009201020112012201320142015 Originales De revisión $\quad$ Otros

Figura 1. Artículos sobre diabetes publicados con filiación peruana en revistas científicas indizadas en WoS o Scopus, 1996-2015. 


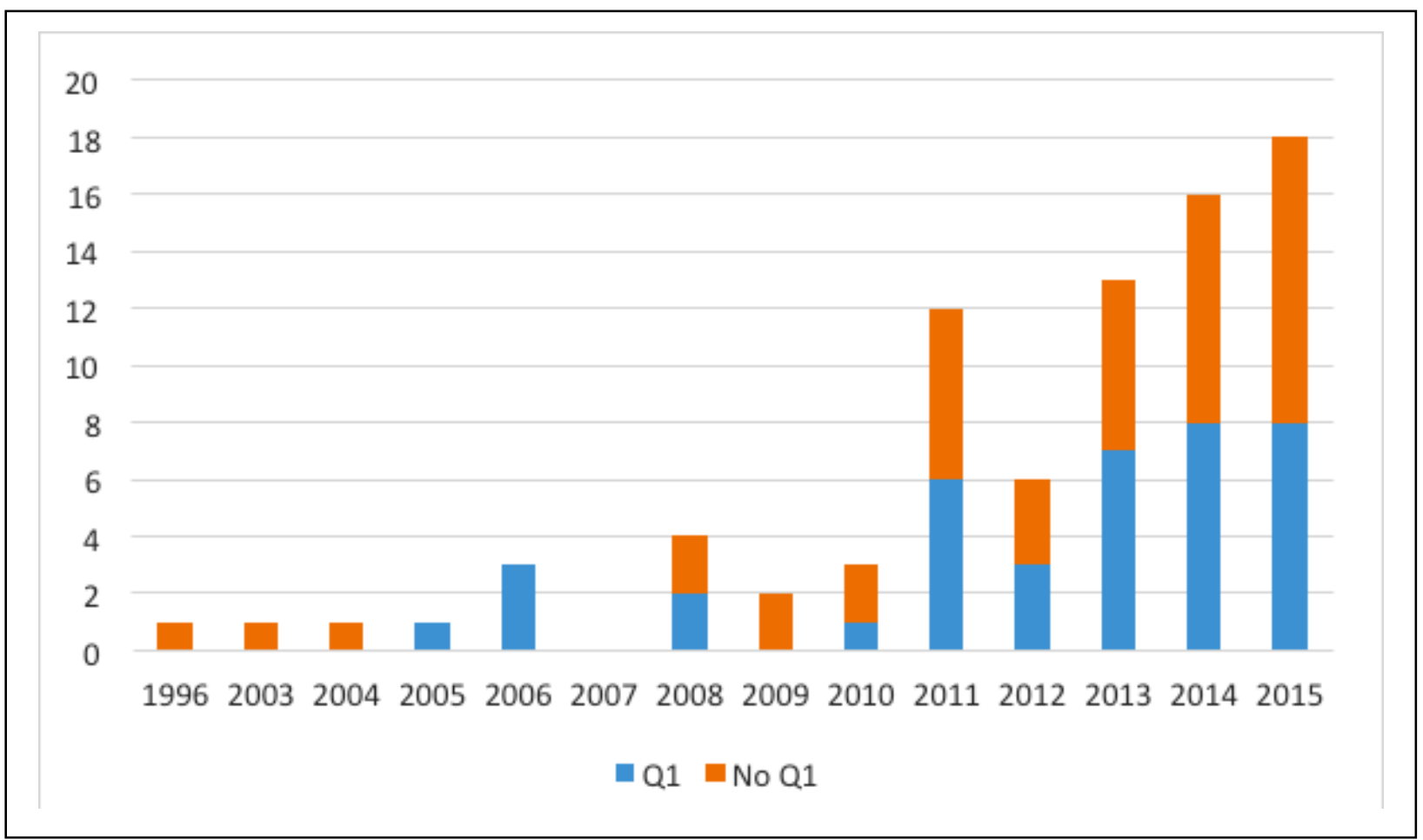

Figura 2. Artículos sobre diabetes publicados con filiación peruana en revistas científicas indizadas en WoS o Scopus, que estén y no estén en el primer cuartil de impacto según SCImago.

\section{DISCUSIÓN}

El presente estudio describe la producción científica peruana en diabetes en el periodo 1996-2005. Se encontró un número bajo pero creciente de publicaciones sobre el tema. Aproximadamente tres cuartos de los artículos fueron originales, entre los cuales el financiamiento fue predominantemente extranjero. La UPCH participó en más de la mitad de los artículos, mientras que la participación de instituciones peruanas ubicadas fuera de la ciudad de Lima es escasa. Aproximadamente la mitad de los artículos tuvieron como autor corresponsal a un autor extranjero. En cuanto a las redes de colaboración, se pueden distinguir cinco conglomerados. La UPCH se sitúa como centro para la colaboración nacional e internacional.

\section{Producción científica}

La diabetes es un problema de salud en Perú, con serias deficiencias en cuanto a su prevención, diagnóstico y manejo. Sin embargo, este impacto a la salud pública no se refleja en el número de artículos publicados. De manera que apenas encontramos 27 artículos publicados durante el periodo 2000-2010, en tanto que un estudio previo realizado en las mismas bases de datos encontró 358 artículos publicados sobre cáncer por autores peruanos en dicho periodo ${ }^{20}$. Asimismo, número de artículos publicados en el país es muy inferior al reportado en periodos de estudio similares en países de la región como Brasil y Argentina ${ }^{8,9}$, además de otros del medio oriente, Nigeria, China e India ${ }^{6-10}$. Lo cual podría deberse a que la producción científica médica en general del Perú es inferior a la de los países comparados ${ }^{31}$.

Esta baja producción científica en diabetes puede estar relacionado a una subestimación de la problemática en el Perú, reflejado en el hecho de que la diabetes no fuera incluida entre las prioridades nacionales de salud de investigación para los años 2010-2014 del Instituto Nacional de Salud del Perú ${ }^{21}$. Aunque debido al creciente interés, este tema es considerado una prioridad para el periodo 2015-202122.

Una limitación importante para realizar investigación en diabetes radica en la falta de presupuesto para investigación en diabetes, puesto que el Consejo Nacional de Ciencia, Tecnología e Innovación Tecnológica (CONCYTEC), que es el principal financista público de la investigación peruana, cuenta con escasos fondos y no prioriza directamente estudios en esta área. Esto explicaría que el financiamiento de entidades extranjeras sea predominante para este tipo de investigación. En este contexto, las entidades encargadas de velar por la salud en el Perú deberán asegurar el financiamiento de investigación en temas prioritarios como diabetes.

En relación al incremento de artículos científicos publicados sobre diabetes en el Perú, es una situación que viene sucediendo en otros países ${ }^{10,23,24}$. Esto puede deberse a una mayor cultura de investigación y publicación en 
TABLA 1

Características de los artículos sobre diabetes publicados con filiación peruana en revistas científicas indizadas en WoS o Scopus, 1996-2015.

\begin{tabular}{|c|c|c|c|}
\hline Característica & $\begin{array}{l}\text { No originales } \\
\mathrm{N}=20 \\
\mathrm{~N}(\%)\end{array}$ & $\begin{array}{l}\text { Originales } \\
N=61 \\
N(\%)\end{array}$ & $\begin{array}{l}\text { Total } \\
\mathrm{N}=81 \\
\mathrm{~N}(\%)^{\prime \prime}\end{array}$ \\
\hline \multicolumn{4}{|l|}{ Idioma del in-extenso } \\
\hline Inglés & $13(65,0)$ & $43(70,5)$ & $56(69,1)$ \\
\hline Español & $2(10,0)$ & $13(21,3)$ & $15(18,5)$ \\
\hline Inglés y español & $4(20,0)$ & $4(6,6)$ & $8(9,9)$ \\
\hline Portugués & $1(5,0)$ & $1(1,6)$ & $2(2,5)$ \\
\hline \multicolumn{4}{|l|}{ Número de instituciones peruanas } \\
\hline 1 & $14(70,0)$ & $30(49,2)$ & $44(54,3)$ \\
\hline 2 & $5(25,0)$ & $18(29,5)$ & $23(28,4)$ \\
\hline 3 o más & $1(5,0)$ & $13(21,3)$ & $14(17,3)$ \\
\hline \multicolumn{4}{|l|}{ Ubicación de las instituciones } \\
\hline Solo instituciones de Lima & $17(85,0)$ & $49(80,3)$ & $66(81,5)$ \\
\hline Solo instituciones de otras ciudades & $3(15,0)$ & $6(9,8)$ & $9(11,1)$ \\
\hline $\begin{array}{l}\text { Instituciones tanto de Lima como de otras } \\
\text { ciudades }\end{array}$ & $0(0,0)$ & $6(9,8)$ & $6(7,4)$ \\
\hline \multicolumn{4}{|l|}{ Colaboración internacional } \\
\hline No & $7(35,0)$ & $21(34,4)$ & $28(34,6)$ \\
\hline Sí & $13(65,0)$ & $40(65,6)$ & $53(65,4)$ \\
\hline \multicolumn{4}{|l|}{ Autor corresponsal } \\
\hline Extranjero & $10(50,0)$ & $30(49,2)$ & $40(49,4)$ \\
\hline Perú & $10(50,0)$ & $31(50,8)$ & $41(50,6)$ \\
\hline Ciudad de Lima & $10(50,0)$ & $26(42,6)$ & $36(44,4)$ \\
\hline UPCH & $5(25,0)$ & $16(26,2)$ & $21(25,9)$ \\
\hline $\mathrm{HNCH}$ & $3(15,0)$ & $1(1,6)$ & $4(4,9)$ \\
\hline UPC & $1(5,0)$ & $2(3,3)$ & $3(3,7)$ \\
\hline Otros Lima & $1(5,0)$ & $7(11,5)$ & $8(9,9)$ \\
\hline Otras ciudades & $0(0,0)$ & $5(8,2)$ & $5(6,2)$ \\
\hline
\end{tabular}

UPCH: Universidad Peruana Cayetano Heredia. $\mathrm{HNCH}$ : Hospital Nacional Cayetano Heredia. UPC: Universidad Peruana de Ciencias Aplicadas

general, junto con un creciente interés en diabetes como problema de salud pública ${ }^{3,25}$.

Estudios de intervención

Un meta-análisis que evaluó intervenciones para mejorar la atención de pacientes con diabetes encontró que en siete de doce estudios la hipoglicemia fue más frecuente en el grupo de intervención que en el grupo control, demostrando el peligro de implementar estrategias que no han sido evaluadas ${ }^{26}$. Sin embargo, nuestro estudio no encontró estudios que hayan evaluado intervenciones de salud pública para mejorar el diagnóstico o manejo de la diabetes, lo cual sugiere que las intervenciones sobre diabetes se están implementando sin la realización previa de estudios rigurosos.

Esto puede deberse a carencias presupuestales o a la escases de investigadores locales entrenados en la realización de este tipo de estudios ${ }^{27}$. Sin embargo, para nuestro periodo de estudio el "Registro Peruano de Ensayos Clínicos" del Instituto Nacional de Salud registra 161 estudios bajo el término "diabetes" 28 , lo cual puede estar reflejando la realización de estudios que finalmente no son publicados en revistas indizadas. Las barreras para la publicación de estos estudios deben ser evaluadas para asegurar la generación y difusión eficiente de este tipo de evidencia.

Análisis de autores y filiaciones

Pocos artículos tuvieron participación de autores peruanos fuera de la ciudad de Lima. Este marcado centralismo de la investigación biomédica en Perú ha sido descrito anteriormente $20,29-33$, e impide la generación de evidencias en las diferentes regiones del Perú, que requieren soluciones acordes a su contexto. 
Figura 3. Análisis de colaboración de los artículos sobre diabetes publicados con filiación peruana en revistas científicas indizadas en WoS o Scopus, 1996-2015.
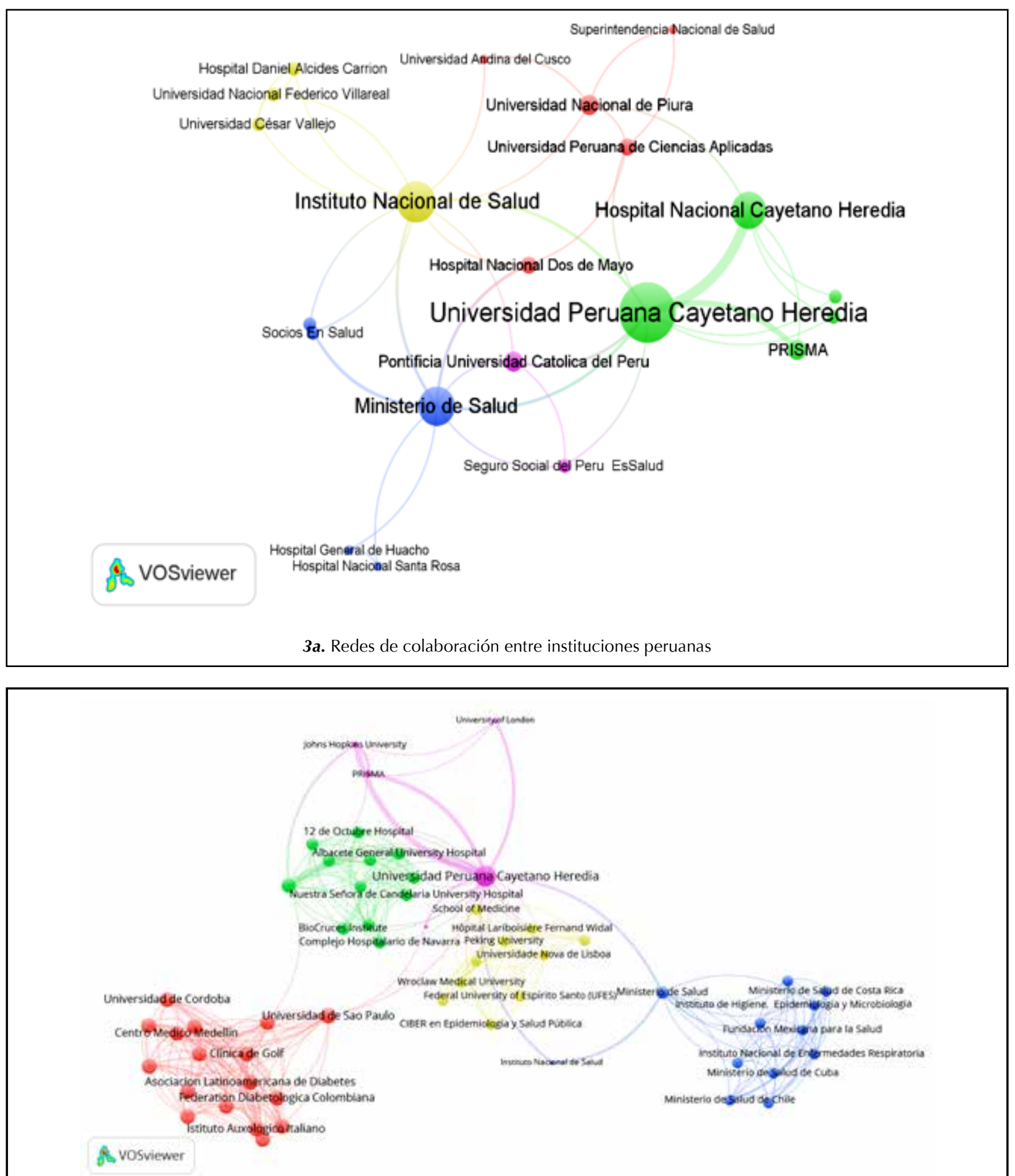

3b. Conglomerados de colaboración entre instituciones peruanas y extranjeras. 
La UPCH es la institución con mayor producción científica en diabetes. Análisis bibliométricos previos realizados a nivel nacional sobre $\mathrm{VIH} / \mathrm{SIDA}^{30}$, tuberculosis ${ }^{33}$, cáncer $^{20}$ y trastornos mentales ${ }^{32}$, mostraron resultados similares.

Aproximadamente la mitad de los artículos tuvieron como corresponsal a un autor con filiación peruana. En concordancia, el 30,4\% de los artículos peruanos en WoS tuvieron como corresponsal a un autor con filiación peruana $^{31}$, en tanto que esta cifra varía del $20,8 \%$ al $81,2 \%$ en otros países sudamericanos ${ }^{34}$. Esta participación activa de investigadores extranjeros puede estar relacionado a mayores facilidades de financiamiento para instituciones extranjeras que realizan estudios en Perú ${ }^{35}$.

\section{Colaboración interinstitucional}

La colaboración inter-institucional genera grupos de trabajo productivos, cuyas publicaciones tienen mayor impacto que aquellas producidos por entidades sin colaboración ${ }^{36,37}$. En nuestro estudio, la UPCH resalta como el centro de la colaboración nacional e internacional, lo cual concuerda con lo descrito en otros estudios bibliométricos peruanos $^{20,31,38}$. Otras redes aún incipientes tienen como eje a otras instituciones, como el Instituto Nacional de Salud, el Ministerio de Salud, y la Clínica El Golf. Estas redes deberían ser potenciadas para aumentar los núcleos de producción científica en el tema. Concordantemente, países que han presentado un aumento en la producción de artículos con colaboración internacional a su vez presentaron un aumento general de la producción científica en diabetes, ${ }^{7,9}$.

También resulta importante fomentar la creación de redes que incluyan instituciones peruanas que estén ubicadas fuera de la ciudad de Lima, con la finalidad de formar competencias en investigación en dichas instituciones, lo cual ayudaría a descentralizar la producción científica y producir evidencias en los distintos contextos peruanos. Una interesante iniciativa al respecto es la que realiza CONCYTEC bajo el nombre de "círculos de investigación", que financia propuestas de investigación que sean realizadas en colaboración interinstitucional.

\section{Características de las revistas}

Se observó que el porcentaje de publicaciones en revistas Q1 se ha mantenido casi constante en los últimos cinco años, lo cual sugiere que el aumento de la producción científica observado en los últimos años no está relegado a revistas de baja calidad, sino que parece deberse a un crecimiento armonioso en términos de cantidad y calidad.

Resulta llamativo que un porcentaje tan alto como el $18,5 \%$ de las publicaciones evaluadas se hayan publicado en una sola revista (Revista Peruana de Medicina Experimental y Salud Pública). Este porcentaje es más alto al encontrado en otros estudios bibliométricos en Perú ${ }^{20,30,33}$. Esto podría significar que los grupos de investigación en diabetes en Perú están acostumbrados a publicar en dicha revista, o a que dicha revista tiene un gran interés en publicar artículos sobre diabetes.

\section{Limitaciones}

Se debe tener presente que el presente estudio no evalúa revistas que no estén indizadas en WoS/Scopus, debido a que se buscó evaluar la producción científica en revistas de calidad. Sin embargo, otros estudios pueden haber sido publicados en revistas no indizadas en dichas bases.

\section{CONCLUSIÓN}

La producción científica peruana en diabetes es baja, y cuenta con una gran participación de investigadores y financiamiento extranjeros. Resalta la falta de estudios que evalúen intervenciones en salud pública. La institución con mayor producción científica y en la que se centran las redes de colaboración es la UPCH. Estos resultados Ilaman a la necesidad de incentivar la investigación en diabetes, con énfasis en la generación de evidencias sobre intervenciones en salud pública, y en el apoyo y empoderamiento de instituciones peruanas y redes de colaboración ubicadas fuera de la ciudad de Lima.

Financiamiento. El presente artículo ha sido autofinanciado por los autores

Conflictos de interés. Los autores declaran no tener conflictos de interés con respecto a la publicación del presente artículo

\section{RESUMEN}

Objetivo: describir la producción científica en diabetes en Perú entre 1996 y 2015. Métodos: Estudio bibliométrico. Se identificaron publicaciones científicas sobre diabetes que tuvieran algún autor con filiación peruana, en las bases de datos Web of Science Core Collection (WoS) y Scopus, entre 1996 y 2015. Se extrajeron manualmente las variables de interés. Se presentaron los resultados descriptivos y las redes de colaboración. Resultados: Se encontraron 81 publicaciones, el 75,3\% fueron artículos originales, de las cuales ninguno evaluó intervenciones en salud pública y $60,7 \%$ recibieron financiamiento extranjero. El 55,6\% tuvieron algún autor afiliado a la Universidad Peruana Cayetano Heredia (UPCH), y el 65,4\% algún autor extranjero. Se puedo distinguir cinco conglomerados de redes de colaboración internacional, todos con participación de la UPCH. Conclusión: La producción científica peruana en diabetes ha sido baja, con ausencia de estudios que evalúen intervenciones en salud pública, y con redes de colaboración centradas en la UPCH.

Palabras clave: Diabetes; bibliometría; redes de colaboración; colaboración internacional; financiamiento en investigación.

\section{BIBLIOGRAFÍA}

1. World-Health-Organization. Global report on diabetes. 2016.

2. Association AD. Statics about Diabetes 2016 http://www. diabetes.org/diabetes-basics/statistics/?referrer=https://www. google.com.pe/. 
3. World-Health-Organization. Diabetes 2016 http://www. who.int/mediacentre/ factsheets/fs312/en/.

4. Royle P, Bain L, Waugh N. Systematic reviews of epidemiology in diabetes: finding the evidence. BMC Med Res Methodol 2005; 5: 2.

5. Arunachalam S, Gunasekaran S, editors. Mapping diabetes research in India and China today. ISSI-2001: International Society for Scientometrics and Informetrics International conference; 2001.

6. Harande YI. Exploring the literature of diabetes in Nigeria: a bibliometrics study. Afr J Diabetes Med 2011; 19(2): 8-11.

7. Rasolabadi M, Khaledi S, Ardalan M, Kalhor MM, Penjvini $S$, Gharib A. Diabetes Research in Iran: a Scientometric Analysis of Publications Output. Acta Informatica Medica. 2015; 23(3): 160-164.

8. Harande YI, Alhaji IU. Basic Literature of Diabetes: A Bibliometrics Analysis of Three Countries in Different World Regions. J Library Information SCl 2014; 2(1): 49-56.

9. Bala A, Gupta B. Diabetes research in India, China and Brazil: A comparative quantitative study, 2000-09. J Health \& Med Informatics 2012; 3: 2.

10. Sweileh WM, Sa'ed HZ, Al-Jabi SW, Sawalha AF. Bibliometric analysis of diabetes mellitus research output from Middle Eastern Arab countries during the period (1996-2012). Scientometrics 2014; 101(1): 819-832.

11. Peykari N, Djalalinia $S$, Kasaeian A, Naderimagham $S$, Hasannia T, Larijani B, et al. Diabetes research in Middle East countries; a scientometrics study from 1990 to 2012. J Res Med Sci 2015; 20(3): 253.

12. Seclen SN, Rosas ME, Arias AJ, Huayta E, Medina CA. Prevalence of diabetes and impaired fasting glucose in Peru: report from PERUDIAB, a national urban population-based longitudinal study. BMJ Open Diabetes Res Care 2015; 3(1): e000110.

13. Ramos W, López T, Revilla L, More L, Huamaní M, Pozo M. Results of epidemiological surveillance of diabetes mellitus in notifying hospitals in Perú. 2012. Rev Peruana Med Exp y Salud Publica 2014; 31(1): 09-15.

14. Taype-Rondan A, Lazo-Porras M, Moscoso-Porras M, Moreano-Sáenz M, Miranda J/. Inadequate glycaemic control in LMIC: health system failures in Peru. Br J Gen Pract 2016; 66(645): 197.

15. Elsevier. Scopus - Content 2016. https://www.elsevier. com/solutions/ scopus/content.

16. Chen H, Ho Y-S. Highly cited articles in biomass research: A bibliometric analysis. Renew Sustain Energy Rev 2015; 49: 12-20.

17. Bayoumy K, MacDonald R, Dargham SR, Arayssi T. Bibliometric analysis of rheumatology research in the Arab countries. BMC Research Notes 2016; 9: 393.

18. Borgatti SP, Everett MG, Freeman LC. Ucinet 6 for Windows: Software for social network analysis, Natick, MA. 2002.

19. Van Eck NJ, Waltman L. Software survey: VOSviewer, a computer program for bibliometric mapping. Scientometrics 2010; 84(2): 523-538.

20. Mayta-Tristán P, Huamaní C, Montenegro-Idrogo IJ, SamanezFigari C, González-A/caide G. Scientific production and cancer-related collaboration networks in Peru 2000-2011: a bibliometric study in Scopus and Science Citation Index. Rev Peru Med Exp y Salud Pública. 2013; 30(1): 31-36.

21. Cabezas C, Yagui M, Caballero P. Priorities for health research in Peru 2010-2014: The experience of a participatory and decentralized process: Systematization of experience.
Lima, Ministerio de Salud, Instituto Nacional de Salud. 2011. http://www.ins.gob.pe/insvirtual/images/otrpubs/ pdf/Prioridades\%20[13.6.11].pdf

22. Instituto Nacional de Salud. National Priorities for Health Research 2015 - 2021. Executive Summary. Lima: Ministerio de Salud, Instituto Nacional de Salud. 2015. http://www. ins.gob.pe/repositorioaps/0/2/jer/mater_prior/Resumen\%20 Ejecutivo\%20Proceso\%20de\%20Prioridades $\% 20$ de $\% 20$ Investigacion\%2011_05_15\%20v4R.pdf

23. Lewin HS. Diabetes mellitus publication patterns, 1984-2005. J Med Libr Assoc 2008; 96(2): 155-158.

24. Geaney F, Scutaru C, Kelly C, Glynn RW, Perry IJ. Type 2 diabetes research yield, 1951-2012: bibliometrics analysis and density-equalizing mapping. PloS one 2015; 10(7): e0133009.

25. International-Diabetes-Federation. IDF Diabetes Atlas - 7th Edition 2015 http://www.diabetesatlas.org/.

26. Shojania KG, Ranji SR, MCDonald KM, Grimshaw JM, Sundaram V, Rushakoff RJ, et al. Effects of quality improvement strategies for type 2 diabetes on glycemic control: a meta-regression analysis. JAMA 2006; 296(4): 427-40.

27. Pérez Jiménez Y, López Flores MA, Abstengo Jorge $Y$. Health scientific production in Ciego de Ávila province between 2003 and 2007. Acimed 1999; 7(2): 115-20.

28. Instituto Nacional de Salud. Peruvian register of clinical trials 2016. http://www.ins.gob.pe/ensayosclinicos/.

29. Pamo Reyna OG. Medical scientific serial publications in Peru. Rev Med Hered. 2005; 16(1): 65-73.

30. Caballero P, Gutiérrez C, Rosell G, Yagui M, Alarcón J, Espinoza M, et al. Bibliometric analysis of scientific production about HIVIAIDS in Peru 1985 - 2010. Rev Peru Med Exp y Salud Publica 2011; 28(3): 470-476.

31. Huamaní C, Mayta-Tristán P. Peruvian scientific production in medicine and collaboration networks, analysis of the science citation index 2000-2009. Rev Peru Med Exp y Salud Publica 2010; 27(3): 315-325.

32. Taype-Rondán Á, Lajo-Aurazo Y, Huamaní C. Peruvian scientific production in SciELO-Peru on mental disorders, 2006-2011. Rev Med Hered 2012; 23(3): 166-171.

33. Yagui-Moscoso $M$, Oswaldo-Jave $H$, Curisinche-Rojas $M$, Gutiérrez C, Romani-Romaní F. Research on tuberculosis in Peru: bibliometric analysis 1981-2010. An Fac Med 2012; 73(4): 299-305.

34. Huamaní C, González G, Curioso WH, Pacheco-Romero J. Scientific production in clinical medicine and international collaboration networks in South American countries ISI Current Contents 2000-2009. Rev Med Chile 2012; 140(4): 466-475.

35. Dahdouh-Guebas F, Ahimbisibwe J, Van Moll R, Koedam $N$. Neo-colonial science by the most industrialised upon the least developed countries in peer-reviewed publishing. Scientometrics 2003; 56(3): 329-443.

36. Gaughan M, Ponomariov B. Faculty publication productivity, collaboration, and grants velocity: using curricula vitae to compare center-affiliated and unaffiliated scientists. Res Eval 2008; 17(2): 103-110.

37. Leta J, Chaimovich H. Recognition and international collaboration: the Brazilian case. Scientometrics 2002; 53(3): 325-335.

38. Huamaní C, Pacheco-Romero J. Scientific collaboration in Peruvian biomedical journals. An Fac med 2011; 72(4): 261-268. 